INTERNATIONAL DESIGN CONFERENCE - DESIGN 2018

https://doi.org/10.21278/idc.2018.0216

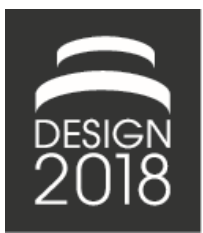

\title{
RESEARCH ON INTELLIGENT DESIGN AND ACCURATE MODELLING OF SPIRAL BEVEL GEARS BASED ON FUNCTION-TO-FORM MAPPING
}

\author{
Z.-G. Xu, K.-Y. Su, J.-F. Zhu and W.-M. Liu
}

\begin{abstract}
A design method of function-to-form mapping is proposed for spiral bevel gear. The rules are established. The mathematical model of tooth surface of the spiral bevel gear is deduced, the boundary of the tooth surface is determined. The intelligent design system of spiral bevel gears is developed on a commercial CAD software SINOVATION. The surface deviation method is used to test the solid model of the spiral bevel gear. The results show that the tooth surface model is accurate, the modeling method are feasible, intelligent and efficient.
\end{abstract}

Keywords: computer aided design (CAD), design methodology, conceptual design, design practice

\section{Introduction}

The design and precise modelling of spiral bevel gears require more intelligent and efficient methods and tools (Sobolewski and Marciniec, 2013). The existing precise modelling methods include virtual machining (Li et al., 2007), free surface reconstruction (Hong et al., 2013) and broad sweep of tooth (Li et al., 2013). The virtual machining method is used to simulate the geometric modelling of the bevel gear in three dimensional software by means of Boolean operation through the modelling of the model tool and the model of the bevel gear blank. The free surface reconstruction method is used to calculate the discrete point of the bevel gear tooth surface based on the tooth surface equation (Lin et al., 2014). Three dimensional software is used to reconstruct the tooth surface of bevel gear based on the surface reconstruction of discrete point (Li et al., 2008; Liu and Zhang, 2011; Ding and Adayi, 2015). The bevel gear tooth surface is obtained by the broad sweep method making use of the spherical involute widening form the bevel gear end face (Wang et al., 2014).

Existing free surface reconstruction method make use of the software, like, Matlab, Excel, etc. to calculation the discrete point of tooth surface, the points are input to construct the 3D model, and the reconstruction is complex and low in efficacy. And it is only suitable for geometric modelling, unable to combine with the design process. Current design method and accurate modelling method of spiral bevel gear is not intelligent enough for efficient modelling, so, this paper put forward an intelligent integrated bevel gear design system based on function-to-form mapping method (F-F mapping for short).

\section{Spiral bevel gear design automation based on function-to-form mapping}

Function-to-form mapping is a "top-down" design process, which is composed of function decomposition, function-form mapping and component reconfiguration (Xu et al., 2001). The "topdown" design process is from qualitative design to quantitative design (Yang et al., 2013). 
A schematic diagram of the design process of a spiral bevel gear based on a F-F mapping is shown in Figure 1. The design problem is divided into three domains: the requirement domain, the functional domain and the form domain. The requirement domain contains the requirement features, the functional domain contains the functional requirements, and the form domain contains the structure parameters, the functional surface and the entity model, etc.

According to the user input, after the requirement decomposition, the functional requirements are obtained.

For example, the demand RA (automobile spiral bevel gear) can be mapped to the transmission precision and dynamic stability of the functional domain.

Functions $F R$ can be decomposed into $F R_{1}$ (transmission power) and $F R_{2}$ (positioning function), where, $F R_{1}$ can be decomposed into $F R_{11}$ (transmission ratio) and $F R_{12}$ (power), $F R_{12}$ can be decomposed into $F R_{121}$ (Dynamic stability) and $F R_{122}$ (power).

Functional and functional units are then mapped to the form domain to obtain the structural units.

In the form domain, $D P$ means the spiral bevel gear model, $D P_{1}$ denotes the bevel gear tooth surface, $D P_{2}$ denotes the positioning surface of bevel gear, $D P_{11}$ denotes the tooth number of gear, $D P_{12}$ denotes the modulus of gear, $D P_{121}$ denotes the gear helical angle measurement, $D P_{122}$ denotes the tooth width of gear.

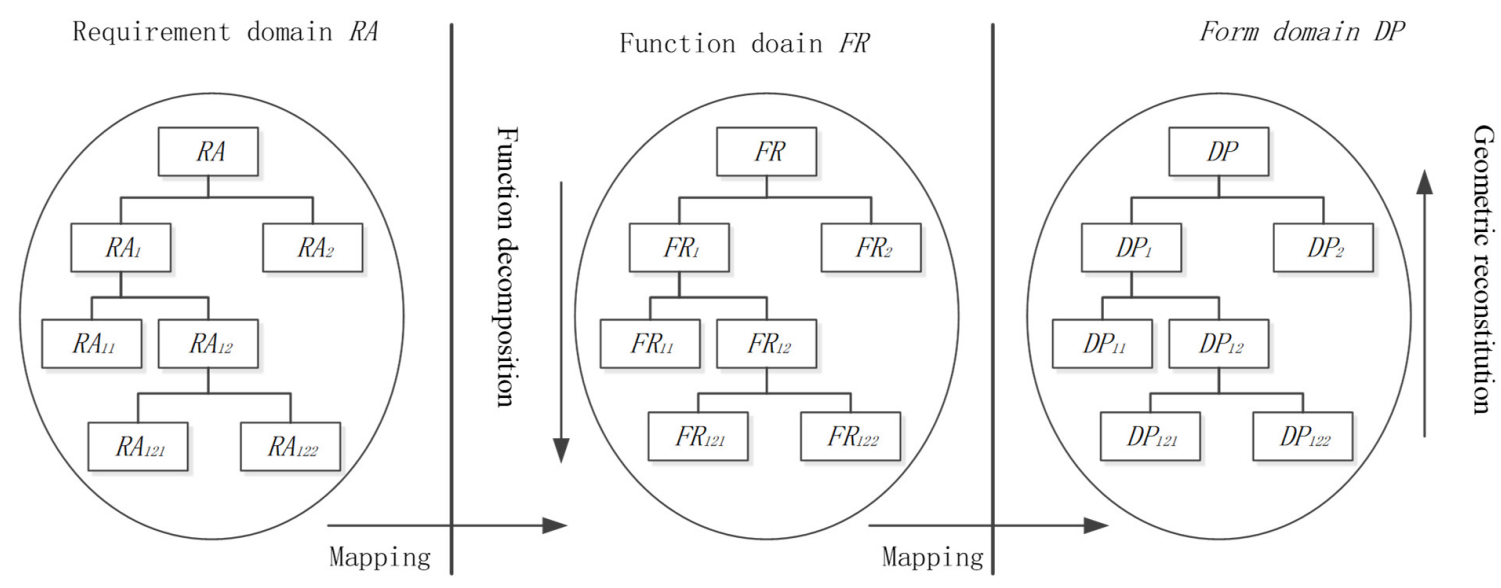

Figure 1. The mechanism of the function-to-form mapping

The design process is the mapping of the function to the structure and the reconfiguration of the entity.

The function-to-form mapping process of the spiral bevel gear design is shown in Figure 2.

Number 1 means function decomposition, number 4,5,6,7,8,9 means the mapping process from subfunctions to sub-forms of the gear. The other numbers denote the parametric design process of the bevel gear. As we notice from Figure 2, the mapping relationship between the function and the structure is complex, and the mapping of the function to the structure is one to one, one to many, and many to many etc. All the mapping rules are obtained from Machinery's Handbook reference (Wen, 2010).

The mapping rules of the spiral bevel gear design are shown in Table 1.

The mapping rule 4 is derived from the strength checking formula of the spiral bevel gear.

The mapping rule 5 is obtained by the polynomial fitting of the line diagram in the mechanical design manual.

Mapping rule 6 and mapping rule 7 are compiled by tables in the mechanical design manual, and the data is stored in the database.

The mapping rule 8 is based on the polynomial fitting, which is based on the requirement of the longitudinal coincidence of the spiral bevel gear transmission.

The mapping rule 9 is derived from the meshing principle of the spiral bevel gear. 


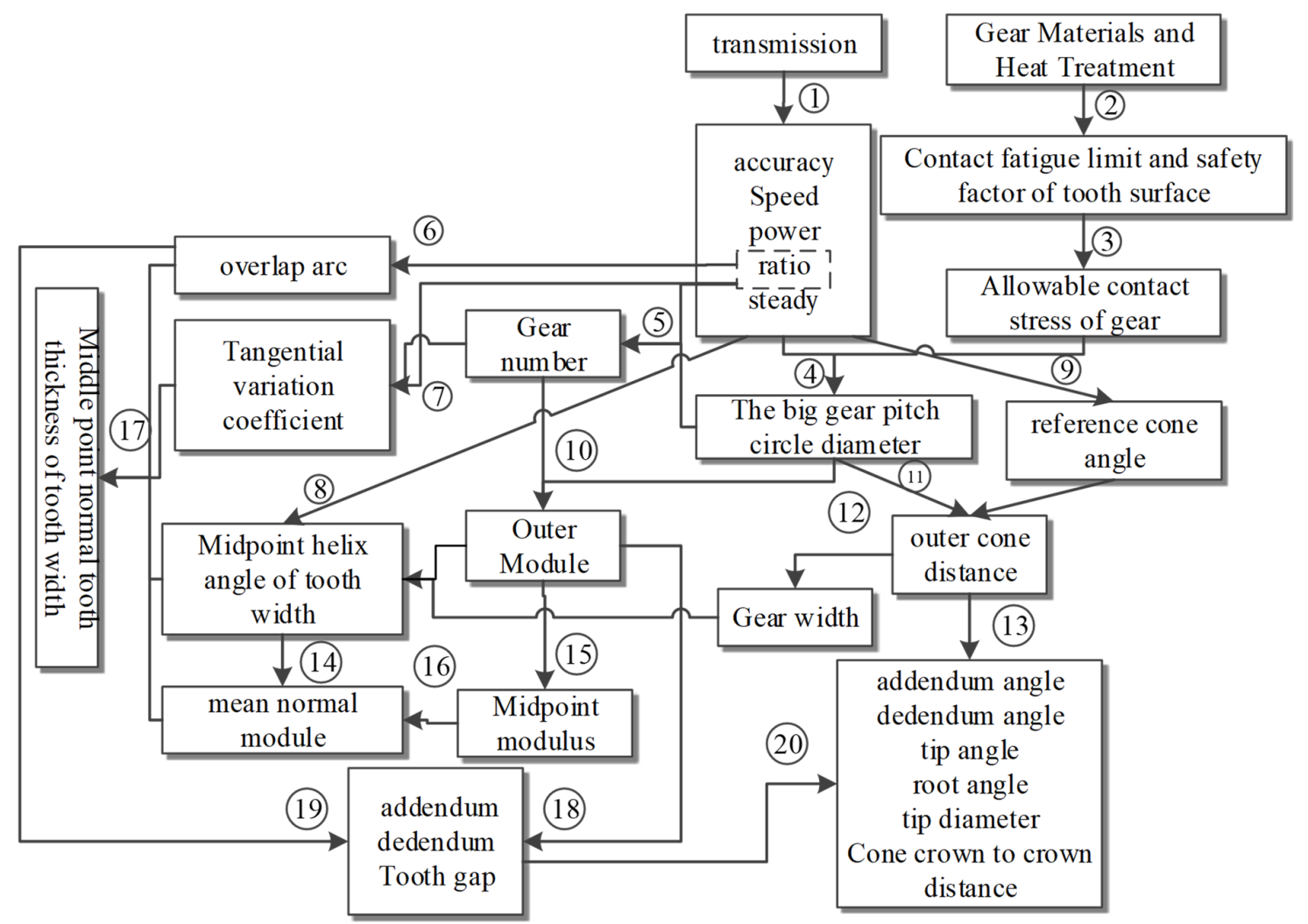

Figure 2. Diagram of function-to-form mapping of spiral bevel gear

Table 1. The rules of function-to-form mapping of spiral bevel gear

\begin{tabular}{|c|c|c|c|}
\hline $\begin{array}{l}\text { Mapping } \\
\text { number }\end{array}$ & Function requirement & Mapping rules & Form parameters \\
\hline 4 & $\begin{array}{l}\text { Accuracy } \mathrm{n} \\
\text { Power P } \\
\text { Ratio } \mu \\
\text { Steady }\end{array}$ & $\begin{array}{c}d_{e 1} \geq 34710 \sqrt[3]{\frac{K P}{n \mu \sigma_{H P}^{2}}} \\
\left(\sigma_{H P} \text { is the allowable contact stress }\right)\end{array}$ & $\begin{array}{l}\text { The big gear pitch circle } \\
\text { diameter } d_{e 1}\end{array}$ \\
\hline 5 & Ratio $\mu$ & $\begin{array}{c}\text { When } 1 \leq \mu \leq 2 \\
Z_{1}=0.0001 d_{e 1}^{2}+0.0157 d_{e 1}+20 \\
\text { When } 2^{2}<\mu \leq 3 \\
Z_{1}=0.0001 d_{e 1}^{2}+0.0227 d_{e 1}+15 \\
\text { When } 3<\mu \leq 4 \\
Z_{1}=0.0002 d_{e 1}^{2}+0.0103 d_{e 1}+12 \\
\quad \text { When } 4<\mu \leq 6 \\
Z_{1}=0.0001 d_{e 1}^{2}+0.0216 d_{e 1}+10 \\
\text { When } 6<\mu \leq 10 \\
Z_{1}=0.0003 d_{e 1}^{2}+0.0001 d_{e 1}+9\end{array}$ & Pinion teeth $Z_{1}$ \\
\hline 6 & Ratio $\mu$ & $x=f_{1}(\mu)$ & Overlap $\operatorname{arc} X$ \\
\hline 7 & Ratio $\mu$ & $x_{t}=f_{2}\left(Z_{1}, \mu\right)$ & $\begin{array}{c}\text { Tangential variation } \\
\text { coefficient } X_{t}\end{array}$ \\
\hline 8 & $\begin{array}{l}\text { Speed n } \\
\text { Power P } \\
\text { Steady }\end{array}$ & $\begin{array}{c}\beta_{m}=85.5594-0.028\left(\mathrm{~b} / \mathrm{m}_{e}\right)^{3} \\
+1.021\left(\mathrm{~b} / \mathrm{m}_{e}\right)^{2}-13.8462 \mathrm{~b} / \mathrm{m}_{e} \\
\beta_{m} \text { convert the force from the pinion } \\
\text { The axial force point to the end }\end{array}$ & $\begin{array}{l}\text { Midpoint helix angle of } \\
\text { tooth width } \beta_{m}\end{array}$ \\
\hline 9 & Ratio $\mu$ & $\delta_{1}=\arctan (1 / \mu)$ & Reference cone angle $\delta_{1}$ \\
\hline
\end{tabular}


After the function-to-form mapped, the main structural parameters of the spiral bevel gear are obtained. The reconfiguration of arc tooth bevel gear for spiral bevel gear is a combination process of free-form surface reconstruction and CSG method.

\section{The mathematical model of the tooth surface of the spiral bevel gear}

The forming process of the tooth surface of the spiral bevel gear is shown in Figure 3, The center of a circular plane coincides with the vertex of the base conic. And the circular plane is tangent to the bottom circle of the base cone. When the circular plane and the base cone are relatively pure rolling, and $\omega / \omega=\sin \delta_{b}$, the trail $P_{1} P_{2}$ formed on a circular plane is a spherical involute. The tooth surface of a bevel gear is thought of being composed of countless $P_{1} P_{2}$.

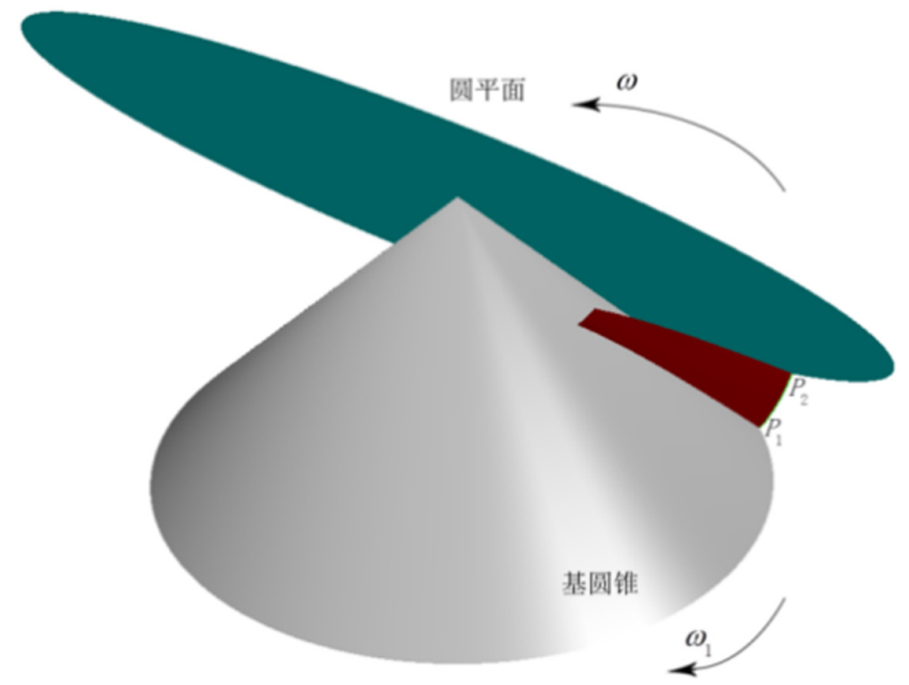

Figure 3. The formation process of tooth surface of spiral bevel gear

The tooth surface equation of the spiral bevel gear (1) - (5) can be derived from the document (Gu et al., 2008).

Where, $R_{\max }$ is the length of the busbar for the base cone, $R_{\max }$ is the base cone angle, $\delta_{b}$ is the base cone angle, $\phi$ is the angle of circular cone bottom circle of spiral bevel gear base, $R_{b i}$ is a busbar length at any point on a conical line. $\eta$ is the angle between the projection of a point in the XY plane and the connection line of the origin to the $\mathrm{X}$ axis. The base cones of the spiral bevel gear are shown in Figure 4.

$$
\left\{\begin{array}{l}
x=A_{1}-B_{1} \\
y=A_{2}+B_{2} \\
z=R_{\max } \cos \delta_{b}-\cos \delta_{b} \cos \left(\phi \sin \delta_{b}\right) R_{b i}
\end{array}\right.
$$

Where,

$$
\begin{aligned}
& A_{1}=R_{b i}\left(\sin \delta_{b} \cos \phi \cos \left(\phi \sin \delta_{b}\right)+\sin \phi \sin \left(\phi \sin \delta_{b}\right)\right) \cos \eta \\
& A_{2}=R_{b i}\left(\sin \delta_{b} \cos \phi \cos \left(\phi \sin \delta_{b}\right)+\sin \phi \sin \left(\phi \sin \delta_{b}\right)\right) \sin \eta \\
& B_{1}=R_{b i}\left(\sin \delta_{b} \sin \phi \cos \left(\phi \sin \delta_{b}\right)-\cos \phi \sin \left(\phi \sin \delta_{b}\right)\right) \sin \eta \\
& B_{2}=R_{b i}\left(\sin \delta_{b} \sin \phi \cos \left(\phi \sin \delta_{b}\right)-\cos \phi \sin \left(\phi \sin \delta_{b}\right)\right) \cos \eta
\end{aligned}
$$




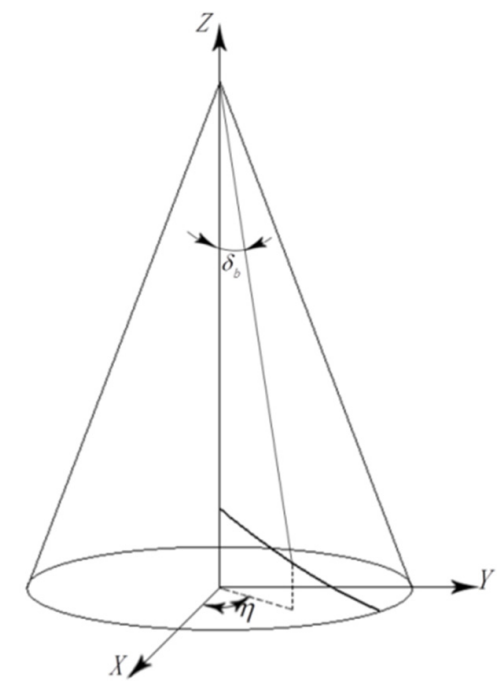

Figure 4. The tooth trace on the cone of spiral bevel gear

On the basis of this, the length of $R_{b i}$ is derived, and the range of the tooth surface of the spiral bevel gear is obtained. The fan-shaped plane of the conical expansion of the bevel gear is shown in Figure 5 . $\theta$ is the polar angle of any point on the line of production, $\mathrm{MN}$ is a shape line, $O_{1}$ is the centre of the shape line, $\beta_{m}$ is the spiral angle of the base cone at the middle point of the tooth width of the spiral bevel gear, $R_{m}$ is the length of the busbar at the middle point of the tooth width, $R_{\min }$ is the length of the busbar at the small end of the gear. According to the characteristics of the shape line of the spiral bevel gear, the Formula (6)-(9) for the polar coordinate equation of the shape line are derived.

$$
R_{b i}=X_{1} \cos \theta+Y_{1} \sin \theta+\sqrt{R_{m}+X_{1} Y_{1} \sin 2 \theta-X_{1}^{2} \sin ^{2} \theta-Y_{1}^{2} \cos ^{2} \theta}
$$

Where,

$$
\begin{aligned}
& \theta=\eta \sin \delta_{b} \\
& X_{1}=\frac{2 R_{m}{ }^{2}\left(1-\sin \beta_{m}\right)+R_{\min }{ }^{2}-R_{m}{ }^{2}}{2 R_{\min }} \\
& Y_{1}=\sqrt{2} R_{m} \sqrt{1-\sin \beta_{m}} * \sin \left(\arccos \left(\frac{2 R_{m}{ }^{2}\left(1-\sin \beta_{m}\right)+R_{\min }{ }^{2}-R_{m}{ }^{2}}{2 \sqrt{2} R_{m} \sqrt{1-\sin \beta_{m}} R_{\min }}\right)\right.
\end{aligned}
$$

$X_{1}$ and $Y_{1}$ are the coordinate value of the centre $O_{1}$ of a shape line.

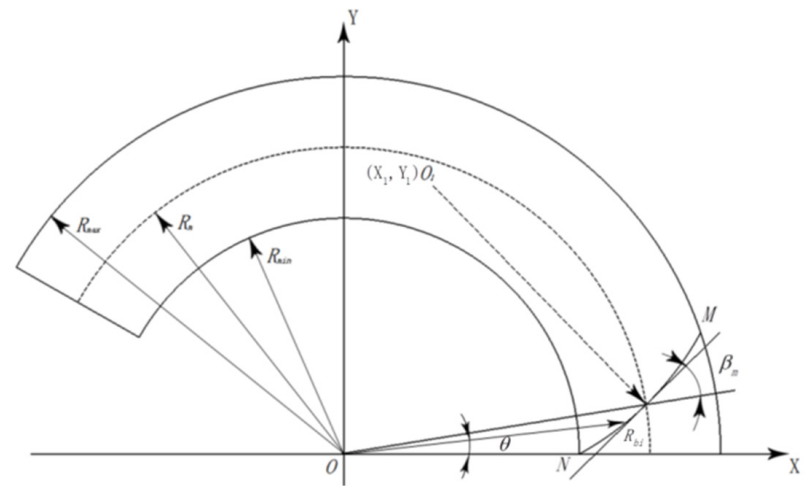

Figure 5. The sectorial plane of base cone of spiral bevel gear 
The range of the tooth surface of the spiral bevel gear decided by $\phi$ and $\eta$ are in the Equation (10)-(13), Where $\delta_{f}$ is Conical angle of spiral bevel gear.

$$
\begin{aligned}
& 0 \leq \phi \leq \arccos \left(\cos \delta_{f} / \cos \delta_{b}\right) / \sin \delta_{b} \\
& 0 \leq \eta \leq\left(C_{1}-C_{2}\right) / \sin \delta_{b} \\
& \mathrm{C}_{1}=\arccos \left(\frac{2 R_{m}^{2}\left(1-\sin \beta_{m}\right)+R_{\min }{ }^{2}-R_{m}{ }^{2}}{2 \sqrt{2} R_{m} \sqrt{1-\sin \beta_{m}} R_{\min }}\right) \\
& \mathrm{C}_{2}=\arccos \left(\frac{2 R_{m}^{2}\left(1-\sin \beta_{m}\right)+R_{\max }{ }^{2}-R_{m}^{2}}{2 \sqrt{2} R_{m} \sqrt{1-\sin \beta_{m}} R_{\max }}\right)
\end{aligned}
$$

\section{Development of intelligent design system for spiral bevel gear}

\subsection{System architecture}

Based on a ADK (Application Development Kit) platform form a Chinese commercial 3D CAD software SINOVATION 7.0, the Visual Studio 2005 and SQL Server 2008 R2 are developing kits. $\mathrm{VC}++$ are the programming language. The architecture of the intelligent design system of spiral bevel gear is shown in Figure 6. The system is composed of function demand module, function to form mapping module, entity reconfiguration module and data management module. Functional requirements modules include functional requirement input and functional requirement decomposition. The mapping modules of function to form include functional form mapping, form unit matching and form scheme generation. The entity reconstruction module includes geometric default reasoning [19], entity geometric correction and entity model output. The data management module includes functional requirement data, structural unit data, power mapping rules, structural scheme data and entity structure data.

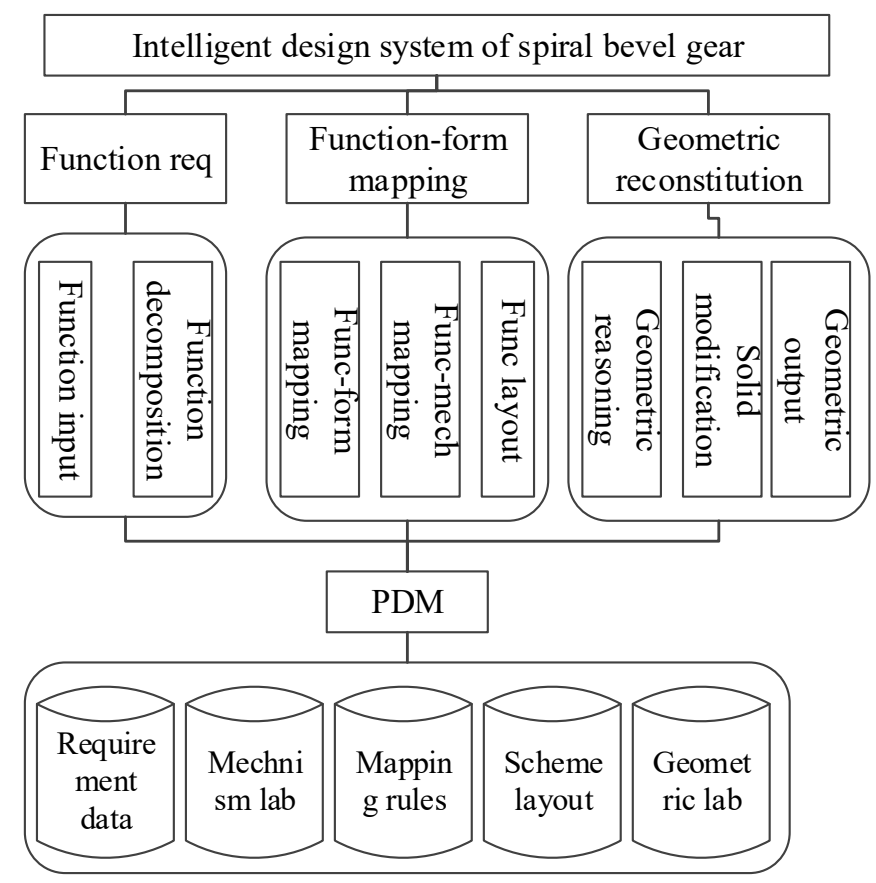

Figure 6. Architecture of intelligent design system of spiral bevel gear 


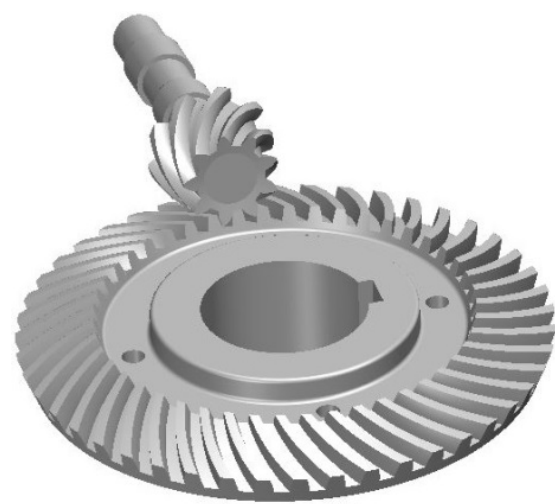

Figure 7. 3D model of the spiral bevel gear model created in SINOVATION

\subsection{Solid reconstruction and precise modelling of spiral bevel gear}

According to the function requirements of the input spiral bevel gear, form parameter requirements of spiral bevel gears are obtained by the decomposition mapping. The system reconfiguration process based on the structure parameters of the spiral bevel gear is as follows.

The custom class CSBGmodeling is created by ADK, which can be used to parameterize the threedimensional model of spiral bevel gear. The class CSBGmodeling member function contains the tooth surface coordinates calculation function of CalculationPoint (CPOINTArray pointA, double *parameterA), the tooth surface to create a function CurSurfaceModeling (CIDENTArray curveA, double *parameterA), the single tooth model reconstruction function ToothRestru (CIDENTArray toothModel, CIDE NTArray cusurfaceA, double * parameterA), the basic body model is created by the function SolidModeling (CIDENTArray solidmodleA, double *parameterA) and spiral bevel gear model to create function SBGmodeling (CIDENTArray, *parameterA).

According to the Equation (1) - (13), the algorithm flow of the calculation function CalculationPoint (CPOINTArray pointA, double *parameterA) of the coordinate point of the tooth surface is given. The known quantity in the Equation (1) - Equation (13) is calculated according to the structural parameter *parameterA obtained by the work structure mapping. Then the independent variable $\phi$ and $\eta$ is divided into discrete values. Then the coordinate value pointA of the discrete point of the tooth surface is obtained by the discrete value (1) - (13), and the discrete point of the tooth surface is created as shown in Figure 8a.

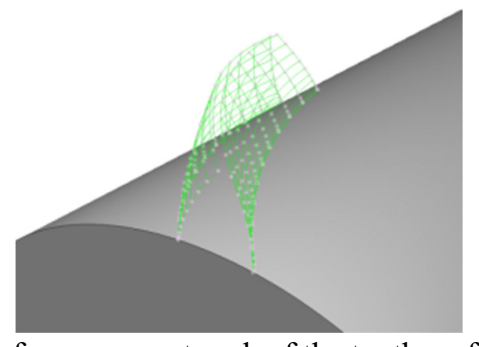

a) The free curve network of the tooth surface

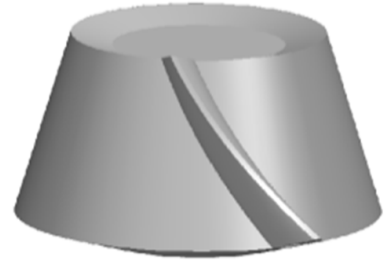

c) Single tooth model

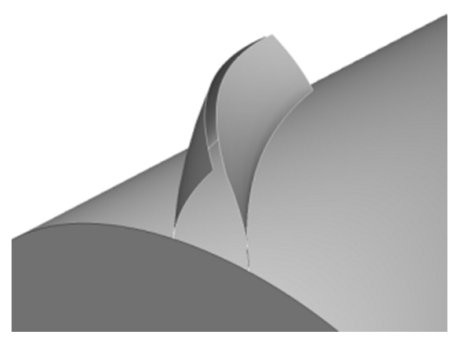

b) Concave convex tooth surface

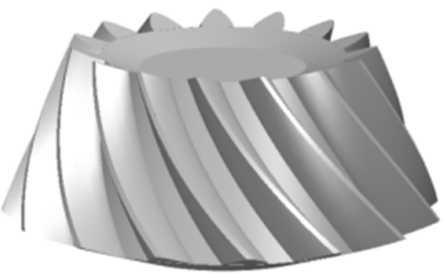

d) Final model

Figure 8. The accurate parameterized modelling process of spiral bevel gear 
Based on the discrete values of the calculated tooth surfaces, the function CurSurfaceModeling (CIDENTArray curveA, double *parameterA) is used to create multiple curve networks and mesh surfaces with multiple points passing through the patches, and the concave surface and the convex tooth surface is created by the tooth surface curveA, as shown in Figure 8b.

On the basis of the tooth surface model, combined with the requirements of gear structure parameters, the tooth top surface is created, the tooth heel and the tooth face are also created. Then the single tooth model with the curveA is reconstructed. The single tooth model is created as shown in Figure 8c.

According to the selected gear structure, the function SolidModeling (CIDENTArray solidmodleA, double * parameterA) is used to create the basic body model, and the partial model of gear shaft is shown in Figure 8c.

Finally, the function SBGmodeling (CIDENTArray SBGA, double *parameterA) is used to array a single tooth model to get the three-dimensional model of spiral bevel gear, as shown in Figure 8d.

\subsection{Test of spiral bevel gear model}

In order to verify the accuracy of the spiral bevel model, the tooth surface deviation method is used to test the deviation of the tooth surface by the vector between the actual tooth point and the theoretical point of the model, as shown in Figure 9a.

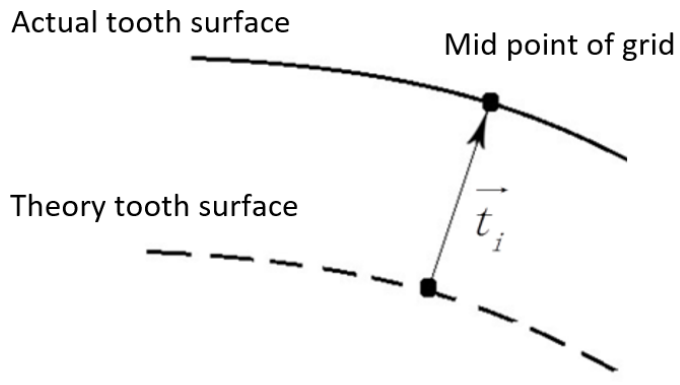

a) Tooth surface deviation vector

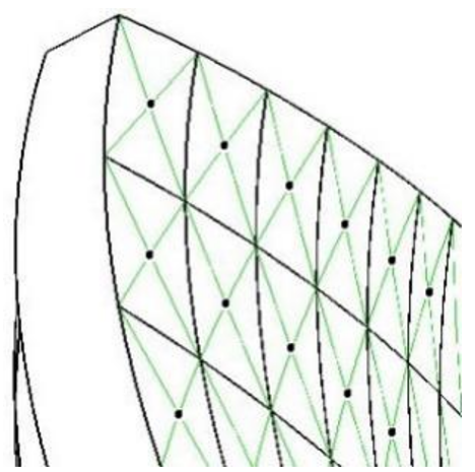

b) Mid-point of gear surface grid

Figure 9. Tooth surface deviation

$\vec{t}_{i}$ is the deviation vector of the ith point on the surface of the tooth, $\left|\vec{t}_{i}\right|$ is the deviation value of the ith point. The midpoint of the 10 meshes of the tooth surface is chosen as the measuring point, as in Figure $9 \mathrm{~b}$. The deviation of the tooth surface after measurement and calculation is shown in Table 2. The deviation value of the tooth surface is kept within a small range.

Table 2. Deviation of mid-point of gear surface grid unit

\begin{tabular}{ccc}
\hline Point i & Deviation vector $\vec{t}_{i} / 10-3 \mathrm{~mm}$ & Deviation value $\left|\vec{t}_{i}\right| / 10-3 \mathrm{~mm}$ \\
\hline 1 & $(-1.128,0.073,2.180)$ & 2.456 \\
2 & $(-0.587,-1.191,4.836)$ & 5.015 \\
3 & $(-0.297,-0.052,3.651)$ & 3.663 \\
4 & $(1.962,3.732,-4.265)$ & 5.997 \\
5 & $(0.144,1.985,-4.048)$ & 4.510 \\
6 & $(-0.119,-1.553,1.813)$ & 2.390 \\
7 & $(-0.421,-2.107,2.952)$ & 3.651 \\
8 & $(0.984,0.412,-2.505)$ & 2.722 \\
9 & $(0.494,4.029,-3.536)$ & 5.384 \\
10 & $(1.754,3.006,-3.397)$ & 4.863 \\
\hline
\end{tabular}




\section{Conclusions}

The existing accurate modelling method of spiral bevel gear is complex and complicated, for this reason, the design method of spiral bevel gear based on the mechanism of reactive function-to-form mapping is proposed. Different from the existing design method of spiral bevel gear, the designer only needs to pay attention to the function requirements of the spiral bevel gear. The form design of the spiral bevel gear can be completed by using the function-to-form mapping rules. This design method has the advantages of top-down, high design efficiency, simple in system development etc. so the parameterized and accurate modelling method of spiral bevel gear combined with free-form surface reconstruction and CSG is proposed. The tooth surface equation and the tooth surface range of the spiral bevel gear are derived, and the spiral bevel gear model with different structures are quick and accurate in solid reconfiguration.

Based on the proposed theory, the intelligent design system of the spiral bevel gear is developed, and the intelligent design and rapid and accurate modelling of the spiral bevel gear are realized. The spiral bevel gear model is tested by the method of tooth surface deviation. The result shows that the error of gear surface modelling is small. The design theory and modelling method proposed are practical, simple and practical.

\section{Acknowledgment}

The above-mentioned research work is supported by the Science, Technology and Innovation Commission of Shenzhen Municipality, JCYJ20160510165328965 and the Chinese NSFC, 61272017, P.R. China, and the National Demonstration Center for Experimental Mechanical Engineering Education (Shandong University).

\section{References}

Ding, H. and Adayi, X. (2015), "Theory of forming spherical involute tooth surface and its accurate cubic NURBS fitting method", Journal of Central South University (Science and Technology), Vol. 46 No. 6, pp. 2052-2058.

Gu, F.-M., Zhou, L., Duan, J.-Z. and Wang, C.-X. (2008), “A new theoretical algorithm for spiral bevel gear", Machinery Design \& Manufacture, Vol. 28 No. 12, pp. 12-13.

Hong, Z.-B., Yang, Z.-J., Zhang, X.C. and Wang, B.-C. (2013), "Milling simulation analysis of spiral bevel gear based on tooth generating line", Journal of Jilin University (Engineering and Technology Edition), Vol. 41 No. 2, pp. 334-339.

Li, G., Cai, Y. and Yan, B. (2013), "Study on the Simulation Technology of Spiral Bevel Gear Machining", Journal of Mechanical Transmission, Vol. 20 No. 4, pp. 37-40.

Li, J.-C., Wang, T.-Y., He, G.-Y., Zheng, H.-J. and Fan, S.-B. (2008), “3D solid accurate modelling of spiral bevel gear based on manufacture method and meshing theory", Journal of Jilin University (Engineering and Technology Edition), Vol. 38 No. 6, pp. 1315-1319.

Li, Z., Wang, Y. and Xue, D. (2007), "Modelling and Simulating of Spiral Bevel Gears Based on Actual Cutting Process", Proceedings of the 2007 IEEE International Conference on Automation and Logistics, Jinan, China, August 18-21, 2007, IEEE, pp. 1694-1698. https://doi.org/10.1109/ICAL.2007.4338845

Lin, C., Li, S.S. and Gong, H. (2014), "Design and 3D modelling of orthogonal variable transmission ratio face gear", Journal of Hunan University (Natural Sciences), Vol. 41 No. 3, pp. 49-55.

Liu, Z.Q. and Zhang, M.H. (2011), "Based on Pro/E Construction Method of Spiral Bevel Gear Logarithm", Advanced Materials Research, $\quad$ Vol. $\quad 215, \quad$ pp. $\quad 167-171$. https://doi.org/10.4028/www.scientific.net/AMR.215.167

Sobolewski, B. and Marciniec, A. (2013), "Method of spiral bevel gear tooth contact analysis performed in CAD environment", Aircraft Engineering and Aerospace Technology, Vol. 85 No. 6, pp. 467-474. https://doi.org/10.1108/AEAT-11-2012-0207

Wang, Y., Adayi, X. and Ding, H. (2014), "Precise Parametric Modelling of Spiral Bevel Gear with Spherical Involute based on UG/Open Platform”, Journal of Mechanical Transmission, Vol. 21 No. 1, pp. 50-54.

Wen, B.C. (2010), Mechanical Design Manual - Volume 2: Mechanical Components Design (Connection, Fastening and Transmission), Machinery Industry Press.

Xu, Z.G., Zhou, Y. and Liu, Q.A.X. (2001), "Theory of Product Creative Design Automation and CAD Modelling”, China Mechanical Engineering, Vol. 12 No. 8, pp. 900-902. 
Yang, B., Gao, C., Yin, X., Wang, X. and Yao, K. (2013), "Extended Product Function Modelling for Conceptual Design”, Journal of Mechanical Engineering, Vol. 49 No. 15, pp. 153-162. https://doi.org/10.3901/JME.2013.15.153

Dr. Zhi-Gang Xu, Professor

Shandong University, Mechanical Engineering

17923, Jingshi Road, Lixiaqu, Wendongxiqu, Jinan, Shandong, 250061 Jinan, China

Email: zhgxu@sdu.edu.cn 